\title{
Editor's Message to Special Issue of Applications and the Internet in Conjunction with Main Topics of COMPSAC 2013
}

\author{
MOTONORI NAKAMURA $^{1, a)}$
}

It is our great pleasure to have the special issue of "Applications and the Internet in Conjunction with Main Topics of COMPSAC 2013" in the IPSJ Journal of Information Processing (JIP). This special issue is successor of the special issue related to the IEEE/IPSJ International Symposium on Applications and the Internet (SAINT), which was started in 2001 as a memorial project of IPSJ's 40th anniversary. The SAINT was incorporated into the Annual International Computer Software \& Applications Conference (COMPSAC) in 2013 and 4 out of 21 tracks took over topics such as networking, computing technologies, advanced applications and services which had been covered by the SAINT. COMPSAC 2013 was held in Kyoto, Japan on July 22-26, 2013 and it was technically sponsored by IPSJ. This special issue was planned to publish articles which are based on the papers presented mainly in the COMPSAC 2013.

We received many submissions related to the Internet and its applications. Among 13 submissions, 9 of them were from authors of papers presented at COMPSAC 2013 including workshops. Out of 13 submissions, 7 papers were accepted (i.e., 54\% acceptance rate). In addition, we had three invited papers from major committee members of COMPSAC 2013. First paper is by Prof. Axel Kupper at Technical University of Berlin, Germany who served as a COMPSAC 2013 program co-chair, and second paper is by Prof. Sahra Sedigh at Missouri University of Science \& Technology who also served as a COMPSAC 2013 program co-chair. Third paper is by Prof. Katsuyuki Yamazaki at Nagaoka University of Technology who was a member of COMPSAC steering committee. Consequently, we had 10 papers published in this special issue.

Finally, I deeply appreciate great efforts and contributions made by all editors of this special issue.

\section{The Editorial Committee}

- Editor in-Chief:

Motonori Nakamura (NII, Japan)

- Editorial Board:

Eiji Kawai (NICT, Japan)

- Editorial Committee:

Ying Cai (Iowa State Univ., USA)

Katsuyoshi Iida (Tokyo Institute of Tech., Japan)

Hiroyuki Ohsaki (Osaka Univ., Japan)

Ryozo Kiyohara (Kanagawa Institute of Tech., Japan)

Hiroyuki Sato (The Univ. of Tokyo, Japan)

Masahiro Jibiki (NICT, Japan)

Takuo Suganuma (Tohoku Univ., Japan)

Hiroki Takakura (Nagoya Univ., Japan)

Kazutoshi Fujikawa (NAIST, Japan)

Kenji Fujikawa (NICT, Japan)

Shigeru Miyake (Hitachi, Japan)

Kenichi Yoshida (Univ. of Tsukuba, Japan) 